\title{
miR-886-3p upregulation in clear cell renal cell carcinoma regulates cell migration, proliferation and apoptosis by targeting PITXI
}

\author{
ZUHU YU ${ }^{1-3^{*}}$, DUQUN CHEN ${ }^{1-3^{*}}$, ZHENGMING SU $^{1,2}$, YIFAN LI $^{1-3}$, WENSHUI YU $^{1,2}$, QIANG ZHANG $^{1-3}$, \\ LIHUA YANG ${ }^{2}$, CAILING LI $^{2}$, SHANGQI YANG ${ }^{1,2}$, LIANGCHAO NI $^{1,2}$, \\ YAOTING GUI ${ }^{2}$, ZEBIN MAO $^{4}$ and YONGQING LAI ${ }^{1,2}$
}

\begin{abstract}
${ }^{1}$ Department of Urology, Peking University Shenzhen Hospital; ${ }^{2}$ The Guangdong and Shenzhen Key Laboratory of Male Reproductive Medicine and Genetics, Institute of Urology, Shenzhen PKU-HKUST Medical Center, Shenzhen 518036;

${ }^{3}$ Anhui Medical University, Anhui, Hefei 230032; ${ }^{4}$ Department of Biochemistry and Molecular Biology, Peking University Health Science Center, Beijing 100083, P.R. China
\end{abstract}

Received March 24, 2014; Accepted August 28, 2014

DOI: $10.3892 /$ ijmm.2014.1923

\begin{abstract}
R-886-3 p$ has been discovered to be involved in the oncogenesis, progression and metastasis of several types of human cancer. The aim of the present study was to identify the biological function of $m i R-886-3 p$ in clear cell renal cell carcinoma (ccRCC) and to determine its possible molecular mechanisms. $m i R-886-3 p$ was found to be significantly upregulated in ccRCC tissues $(\mathrm{P}<0.05)$, in accordance with a previous sequencing result. Functional experiments revealed that forced downregulation of $m i R-886-3 p$ significantly inhibited cellular migration, suppressed cell proliferation and induced cell apoptosis of renal cancer cells. Paired-like homeodomain 1 (PITX1), which has been identified as a tumor suppressor, was found to be downregulated in ccRCC tissues and identified as a target gene of $m i R-886-3 p$. Further experiments demonstrated that the protein level, and not the mRNA level, of PITX1 was significantly decreased or increased when $m i R-886-3 p$ was upregulated or downregulated, respectively, indicating that $m i R-886-3 p$ acted as an oncogene by directly regulating the protein expression of PITX1 at a post-transcriptional level. In conclusion, this study revealed that $m i R-886-3 p$
\end{abstract}

Correspondence to: Professor Yongqing Lai, Department of Urology, Peking University Shenzhen Hospital, Institute of Urology, Shenzhen PKU-HKUST Medical Center, 1120 Lianhua Road, Shenzhen 518036, P.R. China

E-mail: yqlord@163.com

Professor Zebin Mao, Department of Biochemistry and Molecular Biology, Peking University Health Science Center, 38 Xueyuan Road, Beijing 100083, P.R. China

E-mail: zbmao@bjmu.edu.cn

*Contributed equally

Key words: miR-886-3p, clear cell renal cell carcinoma, paired-like homeodomain 1 was upregulated in ccRCC and was involved in cellular migration, proliferation and apoptosis of renal cancer cells by directly targeting the tumor suppressor gene, PITX1.

\section{Introduction}

Renal cell carcinoma (RCC), a common malignant tumor in the urinary system with a low rate of early diagnosis and high rate of recurrence, accounts for $\sim 90 \%$ of renal neoplasms $(1,2)$. One-third of RCC patients have distant metastasis when initially diagnosed and $<30 \%$ of patients who underwent radical surgery have tumor recurrence (3). Clear cell RCC (ccRCC), the main histological type of RCC, accounts for $>80 \%$ of all the RCC patients, is more aggressive than other types and has higher rate of metastasis and a poorer prognosis among common renal malignancies $(4,5)$.

Previously, increasing evidence has demonstrated that microRNA (miRNA), a class of small ( 22 nucleotides) endogenous non-coding RNAs, plays a significant role in various biological processes, including cell metabolism, proliferation, differentiation and apoptosis (6,7). Different from the mechanism of small interfering RNA (siRNA), mature miRNAs usually inhibit genes expression at the post-transcriptional level by binding to the 3'-untranslated regions (3'-UTRs) of target mRNAs, leading to mRNA degradation or depressing translation (8). As miRNA plays important roles in the biological activities of living cells, increasing studies have discovered that certain aberrantly expressed miRNAs in various human cancers were involved in the oncogenesis, progression and metastasis (9). Considerable miRNAs have been characterized as oncogenes or tumor suppressors by targeting downstream genes (10-13).

Numerous studies have discovered that $m i R-886-3 p$ was unconventionally expressed in certain cancers $(8,14-17)$. However, the role of $m i R-886-3 p$ in ccRCC has not been characterized. Whether $m i R-886-3 p$ is involved in the initiation, progression and metastasis of ccRCC remains indistinct. Our previous sequencing for miRNA expression in ccRCC tissues revealed that $m i R-886-3 p$ was overexpressed compared to the 
normal kidney tissues (20). Based on these sequencing results, reverse transcription quantitative polymerase chain reaction (RT-qPCR) was performed to quantify $m i R-886-3 p$ levels in ccRCC tissues, as well as functional experiments to evaluate the effects of $m i R-886-3 p$ on cell migration, proliferation and apoptosis. Bioinformatics, immunohistochemistry, western blot analysis and luciferase reporter assay were performed to identify the downstream targets of $m i R-886-3 p$.

Paired-like homeodomain 1 (PITX1), located in human chromosome 5 , has been identified as a tumor suppressor gene in a number of human malignances $(21,22)$. Thus, we wished to identify whether PITX1 is a target of miR-886-3p.

\section{Materials and methods}

Tissue collection. The ccRCC and paired normal tissues were collected from Guangdong (Anhui, Hunan province, China) and written informed consent was obtained from each patient. Fresh tumor and adjacent normal tissues (located $2.0 \mathrm{~cm}$ outside the visible ccRCC lesions) were frozen in liquid nitrogen once dissected, reviewed and classified with hematoxylin and eosin staining. The characteristics of 36 paired tissues used for qPCR of $m i R-886-3 p$ in the study are shown in Table I. The age range of the patients was 20-76 years, with a median age of 53 years. The study was reviewed and approved by the Hospital Ethics Committees (Peking University Shenzhen Hospital, Shenzhen, China).

Cell culture and transfection. The cell lines used in the study were human renal carcinoma 786-O and $\mathrm{ACHN}$, human embryo kidney cell 293T (HEK-293T) and cervical cancer cell line HeLa, cultured in Dulbecco's modified Eagle's medium (DMEM) (Thermo Fisher Scientific, Inc., Waltham, MA, USA) supplemented with $10 \%$ fetal bovine serum, at $37^{\circ} \mathrm{C}$ in a humidified incubator containing $5 \% \mathrm{CO}_{2}$. The level of $m i R-886-3 p$ in cells was down- or upregulated by transfecting the synthesized $m i R-886-3 p$ inhibitor or $m i R-886-3 p$ mimics (GenePharma Co., Ltd., Shanghai, China) into cells using Lipofectamine 2000 (Invitrogen, Carlsbad, CA, USA) according to the manufacturer's instructions. Random sequences (provided by GenePharma) were used as the negative control. The fold changes of $m i R-886-3 p$ were determined by RT-qPCR in 786-O and ACHN cells $24 \mathrm{~h}$ after transfection.

RNA extraction and RT-qPCR. Total RNA of each sample (tissues and cells) was extracted with TRIzol Reagent (Invitrogen) and purified with the RNeasy Maxi kit (Qiagen, Hilden, Germany) according to the manufacturer's instructions. For the detection of $m i R-886-3 p$, miScript reverse transcription (Qiagen) was used to obtain the cDNA templates. The qPCR was performed using the miScript SYBR-Green PCR kit (Qiagen) and $U 6$ was the internal control. For the mRNA detection, the RevertAid First Strand cDNA Synthesis kit (MBI Fermentas, Inc., Burlington, ON, Canada) was used to obtain the cDNA templates and SYBR ${ }^{\circledR}$ Premix Ex Taq ${ }^{\mathrm{TM}}$ II (Tli RNaseH Plus) (Takara Bio Inc., Otsu, Japan) was used for the quantitation. Human GAPDH was used as the reference gene. The primers (Invitrogen) are shown in Table II and qPCR reaction was performed in the LightCycler $^{\circledR} 480$ real-time PCR system (Hoffmann-La Roche, Basel, Switzerland) according
Table I. Clinicopathological characteristics of 36 patients with ccRCC.

\begin{tabular}{lc}
\hline Variables & No. of cases \\
\hline Age, years & \\
$\geq 53$ & 22 \\
$<53$ & 14 \\
Gender & \\
Male & 19 \\
Female & 17 \\
pT-stage & \\
T1 & 17 \\
T2 & 18 \\
T3 and T4 & 1 \\
AJCC clinical stages & \\
I & 17 \\
II & 16 \\
III+IV & 3
\end{tabular}

ccRCC, clear cell renal cell carcinoma. Disease stages of the patients were classified according to the 2009 American Joint Committee on Cancer (AJCC) staging system (32).

to the manufacturer's instructions. The expression levels were calculated using the $\Delta \Delta \mathrm{Ct}$ method (18).

Migration assay. To assess the influence of $m i R-886-3 p$ on the migratory ability of renal cancer cells (786-O and ACHN) in vitro, the wound scratch assay was performed. Approximately $24 \mathrm{~h}$ after seeding in the 12 -well plates, the cells $(\sim 150,000$ cells/well) were transfected with the $m i R-886-3 p$ inhibitor $(80 \mathrm{pmol})$ or negative control $(80 \mathrm{pmol})$ using Lipofectamine 2000 and cultured in DMEM medium without additional fetal bovine serum. A vertical horizontal wound was made using a sterile $10-\mu l$ pipette tip at $5 \mathrm{~h}$ after transfection. Markers were made to allow for observation of cell migration at the same points. Subsequent to rinsing with phosphate-buffered saline (PBS) to remove the floating cells, the adherent cells were cultured in the incubator at $37^{\circ} \mathrm{C}$. At 0 and $24 \mathrm{~h}$ after the wounds were generated, wound widths $(\mu \mathrm{m})$ were measured using a standard caliper. The wound experiments were performed in triplicate and repeated at least three times.

3-(4,5-Dimethylthiazol-2-yl)-2,5-diphenyltetrazolium bromide (MTT) assay. The MTT assay was carried out to measure cell proliferation of the 786-O and ACHN cells at $0,24,48$ and $72 \mathrm{~h}$ after transfection with the $m i R-886-3 p$ inhibitor or negative control. Approximately 5,000 cells were plated in each well of the 96-well culture plates following transfection. At the time of detection, $20 \mu \mathrm{l}$ of MTT ( $5 \mathrm{mg} / \mathrm{ml}$; Sigma, St. Louis, MO, USA) was added to each well and incubated at $37^{\circ} \mathrm{C}$ for $4 \mathrm{~h}$. Subsequently, the MTT medium was replaced by $150 \mu \mathrm{l}$ dimethylsulfoxide. After shaking for $15 \mathrm{~min}$ at room temperature, the optical density (OD) of each well at the wavelength of $490 \mathrm{~nm}$ was measured using the enzyme immunoassay instrument (Model 680; Bio-Rad, Hercules, CA, USA). 
Table II. Primers for RT-qPCR.

\begin{tabular}{lll}
\hline Name & \multicolumn{1}{c}{ Forward $\left(5^{\prime} \rightarrow 3^{\prime}\right)$} & Reverse $\left(5^{\prime} \rightarrow 3^{\prime}\right)$ \\
\hline$m i R-886-3 p^{\mathrm{a}}$ & CGCGGGTGCTTACTGACCCTT & \\
U6 & CTCGCTTCGGCAGCACA & ACGCTTCACGAATTTGCGT \\
$P I T X 1$ & GTTCAGCGGCCTAGTGCAG & CGGGCTCATGGAGTTGAAGAA \\
GAPDH & AGAAGGCTGGGGCTCATTTG & AGGGGCCATCCACAGTCTTC
\end{tabular}

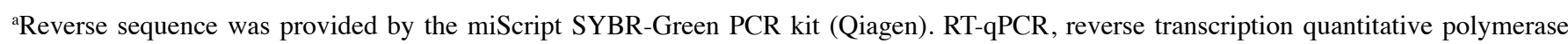
chain reaction; PITX1, paired-like homeodomain 1.

Flow cytometry. Flow cytometry was performed to calculate the apoptosis rates of renal cancer cells transfected with the $m i R-886-3 p$ inhibitor or negative control. The cells (786-O and $\mathrm{ACHN}$ ) were cultured in 6 -well plates at $37^{\circ} \mathrm{C}$ and transfection was conducted at a cell confluence of $\sim 60 \%$. At $48 \mathrm{~h}$ after transfection, adherent and floating cells in each well were harvested and washed twice with cold PBS, resuspended in $1 \mathrm{X}$ binding buffer, and $5 \mu \mathrm{l}$ Annexin V-FITC (Invitrogen) and $10 \mu \mathrm{l}$ propidium iodide were added. Within $30 \mathrm{~min}$ of staining, according to the manufacturer's instructions, the fluorescence of each sample was detected by flow cytometry (Beckman Coulter, Inc., Brea, CA, USA) using 488 nm excitation.

Bioinformatics. The potential targets of $m i R-886-3 p$ were created by combining four public algorithms, which were TargetScan (http://www.targetscan.org/), miRanda (http://www. targetscan.org/), miRWalk (http://www.umm.uni-heidelberg. de/apps/zmf/mirwalk/) and PicTar (http://pictar.mdc-berlin. $\mathrm{de} /$ ). The putative genes that were predicted by at least three algorithms were accepted and the candidates were chosen based on the gene function.

Plasmid construction and luciferase reporter assay. The 3'-UTR of PITX1 (930 base pair) containing putative binding site (5'-CACCCGC-3') for $m i R-886-3 p$ was cloned into the empty psiCHECK-2 Vector (Promega Corporation, Madison, WI, USA), generating the wide type of psiCHECK2-3'UTR (Wt). The mutant type (Mt) was generated by changing the putative binding site to 5'-ACGCCGC-3' in the complementary site for the seed region of $m i R-886-3 p$. All the constructed plasmids were sequenced-verified by DNA sequencing analysis.

For the luciferase reporter assay, HEK-293T and HeLa cells were seeded in 12-well plates, co-transfected with $0.5-\mu \mathrm{g}$ constructed plasmids and 40 pmol $m i R-886-3 p$ mimics or control mimics using Lipofectamine 2000 (Invitrogen). At $24 \mathrm{~h}$ after transfection, firefly and renilla Luciferase of the cells were detected using the Dual-Luciferase Reporter Assay system (Promega) on the Modulus ${ }^{\mathrm{TM}}$ Single Tube Multimode Reader (Bio-Systems International, Beloit, WI, USA). All the wells were performed in triplicate and repeated at least three times.

Immunohistochemistry and western blot analysis of PITX1. The immunohistochemical assay of PITX1 was performed in $20 \mathrm{ccRCC}$ paraffin-embedded tissues according to standard procedures. The $5-\mu \mathrm{m}$ sections were dewaxed in xylene, rehydrated in descending ethanol series, incubated in $3 \%$ hydrogen peroxide solution for $20 \mathrm{~min}$, boiled in $0.01 \mathrm{M}$ citrate buffer ( $\mathrm{pH}$ 6.0) for antigen retrieval and treated in $10 \%$ bovine serum albumin for $30 \mathrm{~min}$ in $37^{\circ} \mathrm{C}$ to block non-specific protein binding. Subsequently, the sections were incubated in diluted (1:100) PITX1 rabbit polyclonal antibody (NBP1-19686; Novus International, Saint Charles, MO, USA) overnight at $4^{\circ} \mathrm{C}$. The sections were rinsed with PBS and treated with the anti-rabbit IHC kit (Maixin Bio, Fuzhou, China) at $37^{\circ} \mathrm{C}$ for $30 \mathrm{~min}$, followed by staining with a DAB kit (Maixin Bio) for 4 min and counterstained with hematoxylin. The negative controls were performed with omission of the primary antibodies.

For western blot analysis, protein was extracted from 16 cases of paired ccRCC tissues and 786-O cells that were transfected with $m i R-886-3 p$ mimics or inhibitors. The samples were homogenised in lysis buffer on ice and the protein concentration was quantified using the Pierce BCA Protein assay kit (Thermo Fisher Scientific). Following separation by $10 \%$ SDS-PAGE and transferring onto nitrocellulose membranes, the protein samples $(100-\mu \mathrm{g})$ were blocked with $10 \%$ skimmed milk at room temperature for $2 \mathrm{~h}$ and incubated in primary antibodies overnight at $4^{\circ} \mathrm{C}$. Primary antibodies of PITX1 and $\beta$-actin (internal control) were rabbit polyclonal anti-PITX1 (1:1,000, NBP1-19686) and rabbit polyclonal anti- $\beta$-actin (1:10,000, NB600-532) (Novus International), respectively. Subsequent to washing three times with Tris-buffered saline with Tween, the membranes were treated with horseradish peroxidase (HRP) AffiniPure goat anti-rabbit immunoglobulin G (H+L) (E030120-01; EarthOx Life Science, Millbrae, CA, USA) for $2 \mathrm{~h}$, followed by detection of the protein bands using the Immun-Star ${ }^{\mathrm{TM}}$ HRP Chemiluminescence kit (Bio-Rad). Each assay was repeated $\geq 3$ times.

Statistical analysis. The data are presented as the means \pm standard deviation and statistical significance was determined with t-test or Mann-Whitney U test, as appropriate. All the statistical analysis was performed with SPSS 17.0 (SPSS, Inc., Chicago, IL, USA) and values of $\mathrm{P}<0.05$ were considered to indicate a statistically significant difference.

\section{Results}

Verification of miR-886-3p upregulation in ccRCC tissues by $q P C R$. By means of miRNA sequencing, previous studies have discovered that $m i R-886-3 p$ was upregulated in ccRCC 

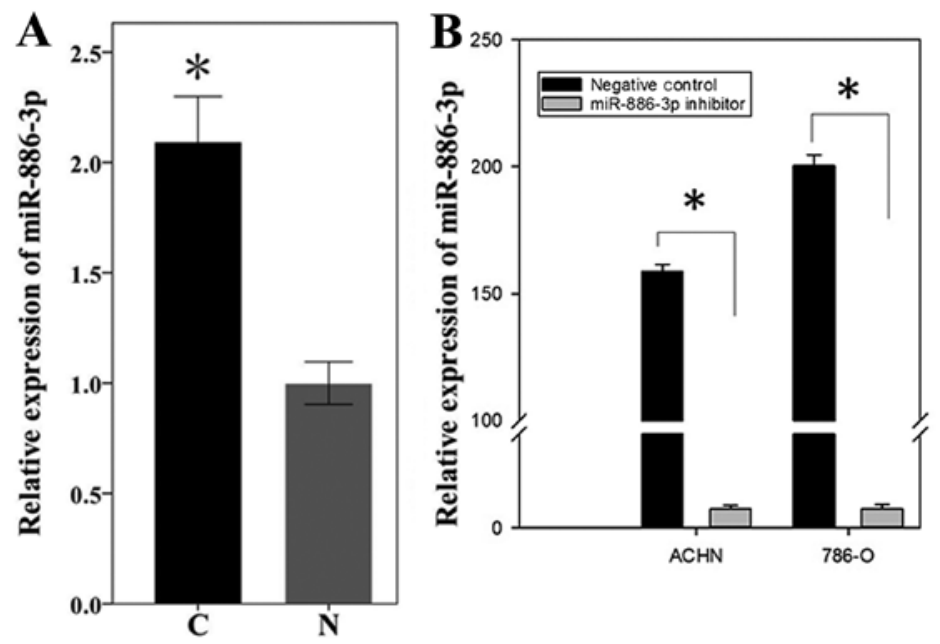

Figure 1. (A) Expression of $m i R-886-3 p$ in 36 paired ccRCC tissues (C) and adjacent normal tissues (N). (B) Relative expression of $m i R-886-3 p$ in ACHN and $786-\mathrm{O}$ cells $24 \mathrm{~h}$ after transfection. ${ }^{*} \mathrm{P}<0.05$. ccRCC, clear cell renal cell carcinoma.

tissues $(19,20)$. To validate the sequencing result, qPCR was used to quantify the miR-886-3p levels in 36 paired ccRCC and adjacent normal tissues. As shown in Fig. 1A, miR-886-3p was significantly higher in ccRCC tissues compared to adjacent normal tissues ( $\mathrm{P}=0.001$, paired-t test), which was identical to the sequencing result.

Downregulation of miR-886-3p inhibits cell migration, proliferation and induces cell apoptosis in vitro. To explore the role of upregulated $m i R-886-3 p$ in biological behavior of renal cancer, the $m i R-886-3 p$ levels in 786-O and ACHN cells were downregulated by transfecting the synthetic miR-886-3p inhibitor (Fig. 1B). The wound scratch assay, MTT assay and flow cytometry were performed. The results of the wound scratch assay showed the wound widths of the experimental group ( $m i R-886-3 p$ inhibitor group) at $24 \mathrm{~h}$ after transfection were much more spacious compared to the negative control group $(\mathrm{P}<0.05)$. In particular, the cells transfected with $m i R-886-3 p$ migrated less distance, prompting the downregulation of $m i R-886-3 p$, which inhibited the migratory ability of the renal cancer cells (Fig. 2).

The MTT assay was used to determine the change of cell proliferation at 24,48 and $72 \mathrm{~h}$ after the level of $m i R-886-3 p$ was downregulated. Compared to the control group, the OD values of the 786-O cells transfected with $m i R-886-3 p$ were decreased by $4.6(\mathrm{P}>0.05), 11.7(\mathrm{P}<0.05)$ and $16.1 \%(\mathrm{P}<0.05)$, whereas the OD values of the ACHN cells were decreased by $7.0(\mathrm{P}>0.05)$, $13.6(\mathrm{P}<0.05)$ and $15.8 \%(\mathrm{P}<0.05)$ at 24,48 and $72 \mathrm{~h}$ after transfection, respectively (Fig. 3). The results demonstrated that abatement of $m i R-886-3 p$ depressed cell proliferation of ccRCC.

To evaluate the effect of reduced $m i R-886-3 p$ expression on renal cancer cell apoptosis, flow cytometry was used to calculate the apoptosis rates of 786-O and ACHN cells following transfection. The results showed that the apoptosis rates of 786-O cells transfected with the $m i R-886-3 p$ inhibitor and negative control groups were 10.7 and $2.5 \%(\mathrm{P}<0.05)$, while the apoptosis rates of the ACHN cells were 12.2 and $4.6 \%(\mathrm{P}<0.05)$, respectively, as shown in Fig. 4. The experiments were repeated three times and identical results were obtained, indicating that the reduction of $m i R-886-3 p$ promoted renal cancer cell apoptosis.
miR-886-3p targets the tumor suppressor gene, PITX1. miRNAs are believed to control gene expression at the post-transcription level by targeting the 3'-UTRs of the downstream genes. To explore the downstream targets of $m i R-886-3 p$, bioinformatics was performed to identify the potential targets. PITX1 was one of the putative genes by three algorithms (TargetScan, miRanda and miRWalk) simultaneously, of which the mRNA contained a complementary site for the seed region of $m i R-886-3 p$ (Fig. 5A). Furthermore, PITX1 has been identified as a tumor suppressor and is associated with the progression and development in various cancers (21-25).

To determine whether PITX1 was directly regulated by $m i R-886-3 p$, the 3'-UTR fragment of PITX1 containing the putative or mutant binding site were cloned into psiCHECK-2 to construct recombinant plasmids (Wt and $\mathrm{Mt}$ ) and the luciferase reporter assay was performed in $293 \mathrm{~T}$ and HeLa cells. As shown in Fig. 5B, the relative luciferase activity of wide-type plasmids containing the putative binding site was significantly decreased when transfected with $m i R-886-3 p$ mimics in $293 \mathrm{~T}$ cells $(\mathrm{P}<0.05)$, whereas no notable reduction was observed in the mutant groups. An identical result was also obtained in Hela cells, indicating that $m i R-886-3 p$ may restrain the expression of PITX1 by targeting the putative binding site in the 3 '-UTR.

Protein expression of PITX1 in ccRCC tissues and adjacent normal tissues. PITX1 was discovered to be downregulated in a number of types of human cancer, including gastric cancer, colorectal carcinoma and lung cancer. However, thus far the protein expression of PITX1 in renal cancer is unclear. Immunohistochemistry and western blot analysis were performed to determine the protein expression of PITX1 in 20 pairs of ccRCC sections and 16 pairs of fresh tissues, respectively. The results of the immunohistochemical assay showed that PITX1 reduction was observed in $90 \%(18 / 20)$ of the ccRCC sections (Fig. 6A), while western blot analysis also showed that PITX1 was downregulated in the ccRCC tissues (Fig. 6B). The association between the expression level of PITX1 and clinicopathological variables was not evaluated due to limited cases. 

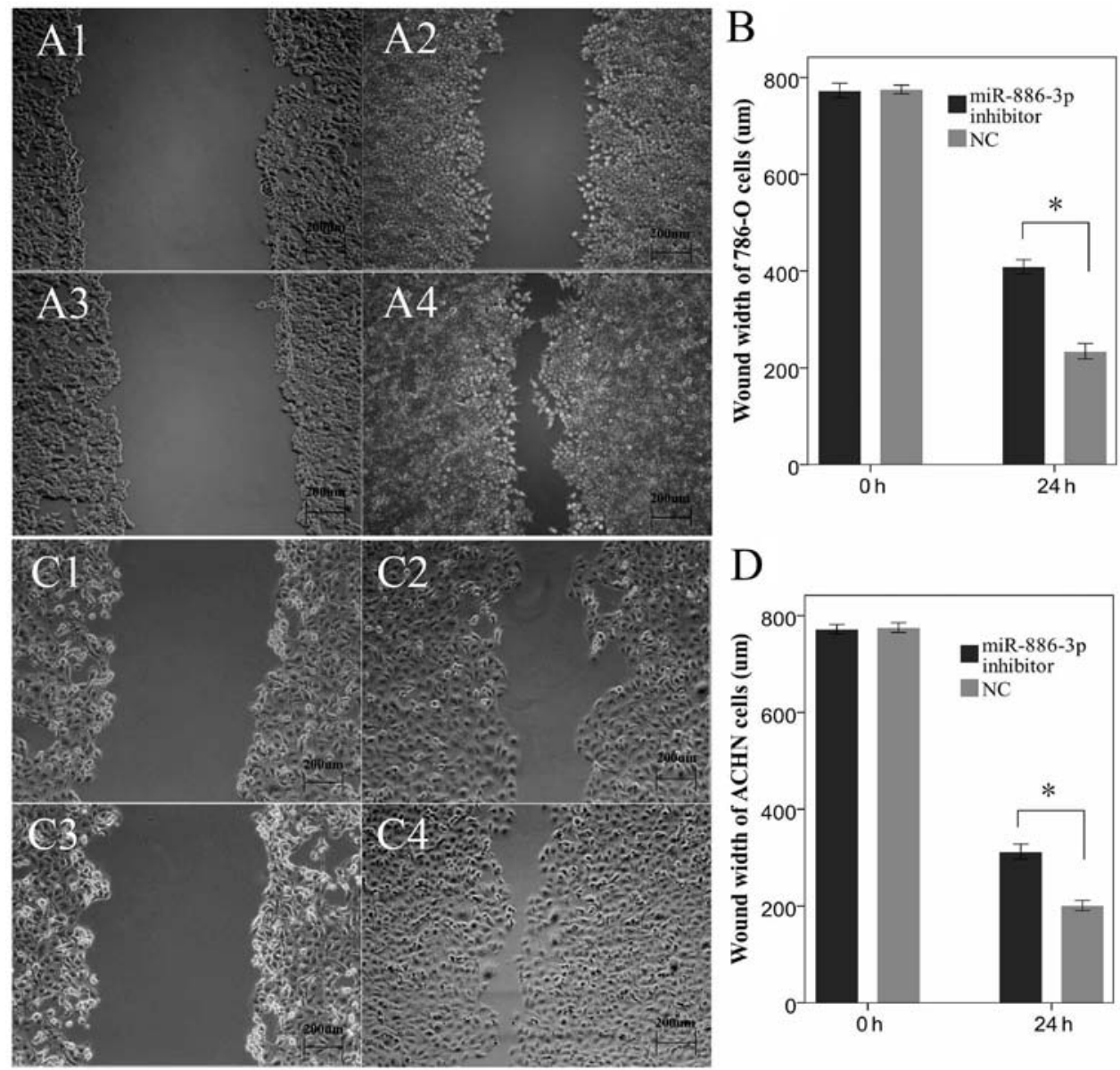

Figure 2. Wound scratch assay for 786-O and ACHN cells $24 \mathrm{~h}$ after transfection. (A1 and A2) Images of the 786-O cells transfected with the miR-886-3p inhibitor at 0 and $24 \mathrm{~h}$ after the wounds were created at the same point. (A3 and A4) Images of the 786-O cells transfected with the negative control (NC) at 0 and $24 \mathrm{~h}$ after the wounds were created. (B) Wound widths $(\mu \mathrm{m})$ of 786-O cells using a standard caliper. $(\mathrm{C} 1$ and $\mathrm{C} 2)$ Images of the ACHN cells transfected with the miR-886-3p inhibitor at 0 and $24 \mathrm{~h}$ after the wounds were created at the same point. (C3 and C4) Images of the ACHN cells transfected with the negative control at 0 and $24 \mathrm{~h}$ after the wounds were created. (D) Wound widths $(\mu \mathrm{m})$ of ACHN. ${ }^{*} \mathrm{P}<0.05$.
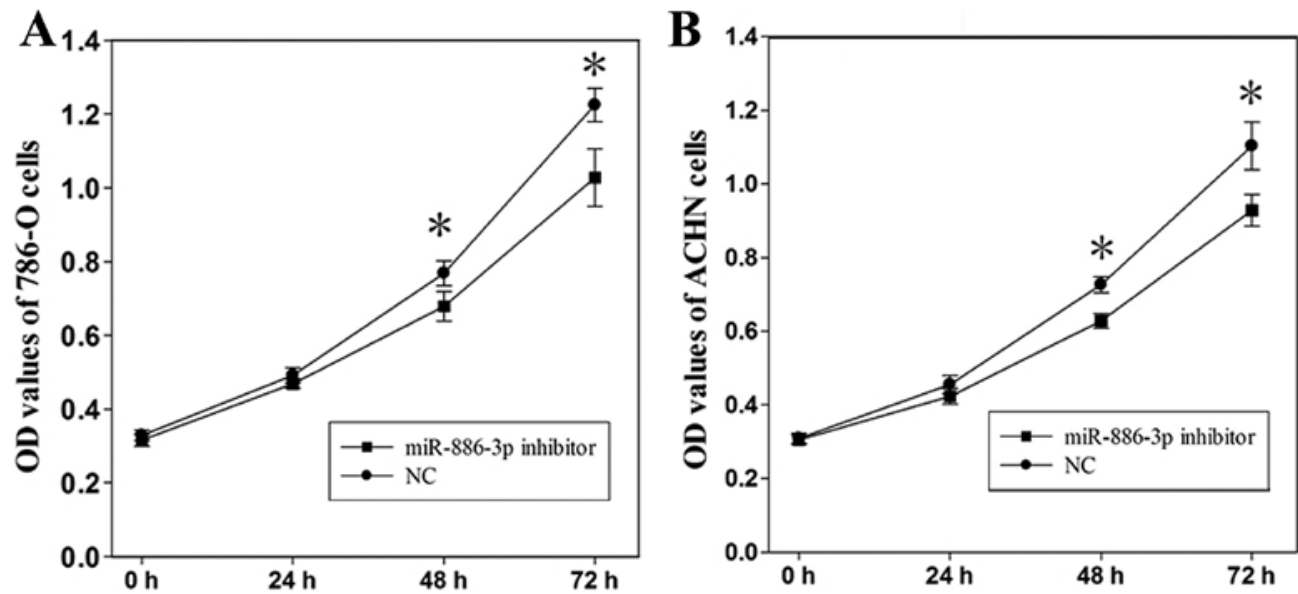

Figure 3. MTT assay for 786-O and ACHN cells transfected with the miR-886-3p inhibitor or negative control (NC). (A) Cell proliferation of 786-O cells and (B) ACHN cells. ${ }^{*} \mathrm{P}<0.05$.

Regulation of PITX1 by miR-886-3p. To validate the results of the luciferase reporter assay and to evaluate the association between PITX1 expression and the $m i R-886-3 p$ level, qPCR and western blot analysis were performed to quantify the mRNA and protein level of PITX1 in 786-O cells $48 \mathrm{~h}$ after transfection with $m i R-886-3 p$ mimics or inhibitor. Notably, the mRNA level of PITX1 in 786-O cells was not significantly decreased or increased following transfection $(\mathrm{P}<0.05$, Fig. 7A). By contrast, the PITX1 protein level was significantly upregulated when 786-O cells were transfected with the $m i R-886-3 p$ inhibitor and 

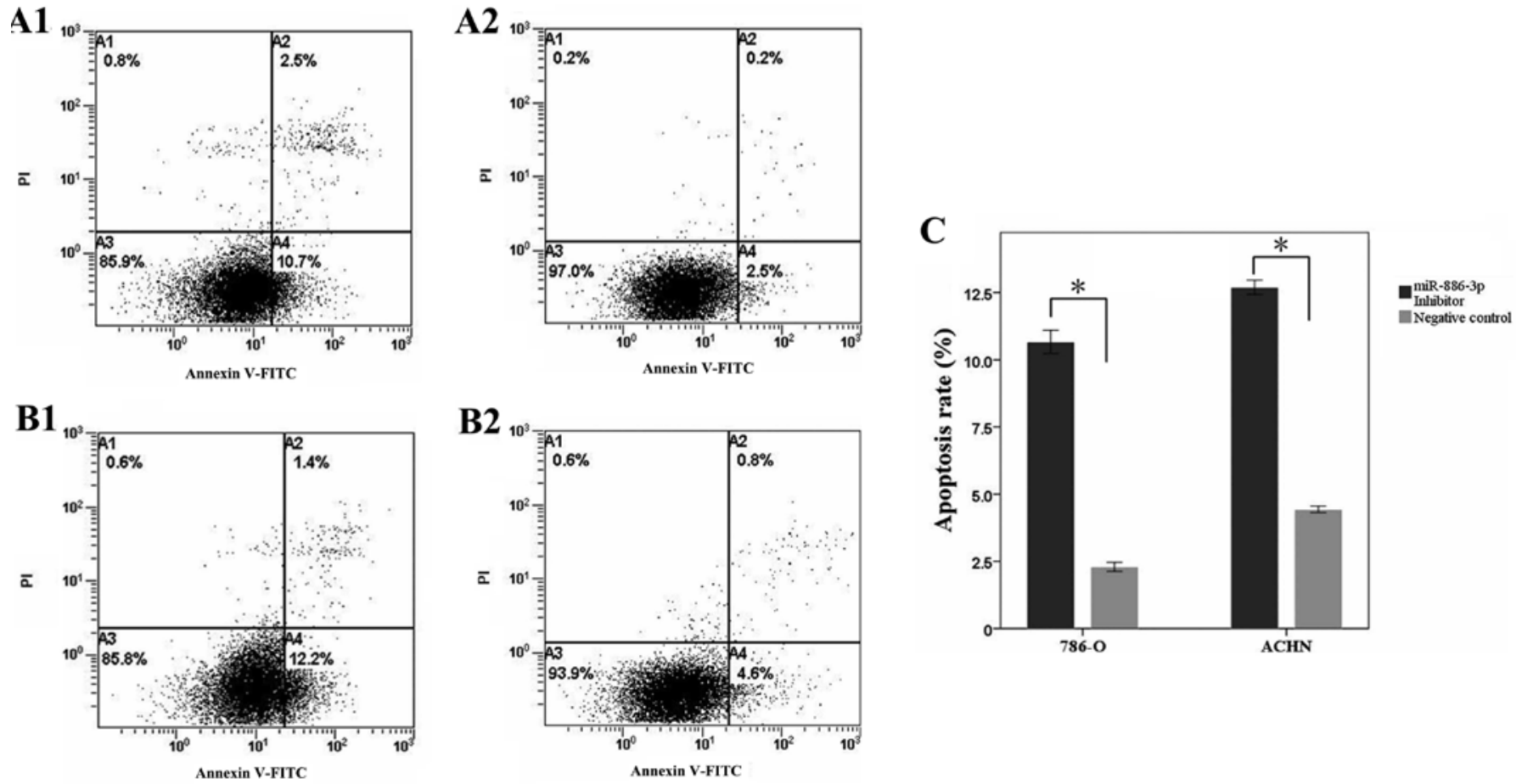

Figure 4. Flow cytometry for 786-O and ACHN cells transfected with the miR-886-3p inhibitor or negative control. (A1 and A2) 786-O cells transfected with the (A1) $m i R-886-3 p$ inhibitor or (A2) negative control. (B1 and B2) ACHN cells transfected with the (B1) miR-886-3p inhibitor or (B2) negative control (C) Apoptosis rates of cells transfected with the $m i R-886-3 p$ inhibitor and negative control. " $\mathrm{P}<0.05$.
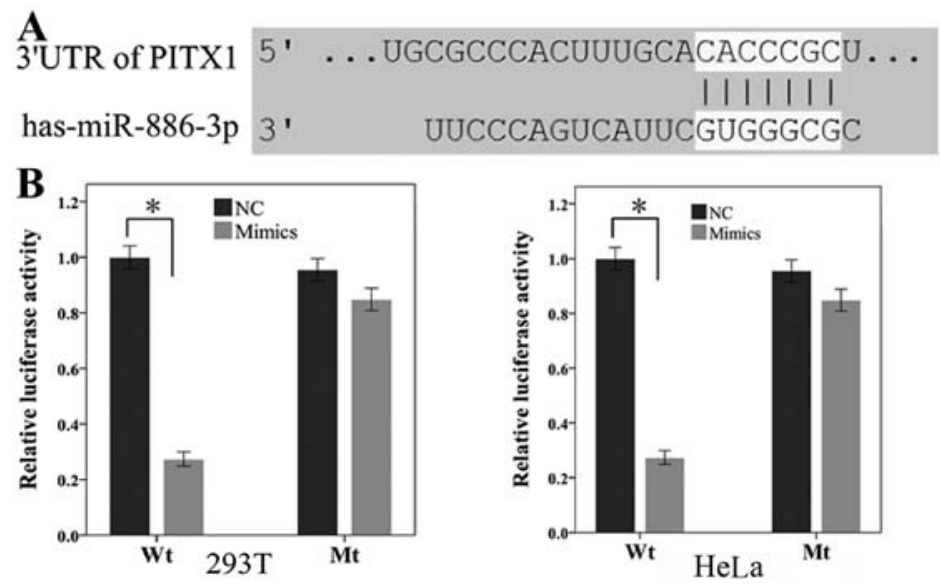

Figure 5. (A) Complementary site for the seed region of $m i R-886-3 p$ predicted by TargetScan. (B) Luciferase reporter assay for 293T and HeLa cells following co-transfection with the constructed plasmids and $m i R-886-3 p$ or control mimics. All Luciferase assays were performed in triplicates and measurements were obtained $24 \mathrm{~h}$ after transfection. UTR, untranslated region; PITX1, paired-like homeodomain 1; Wt, wild type; Mt, mutant type.
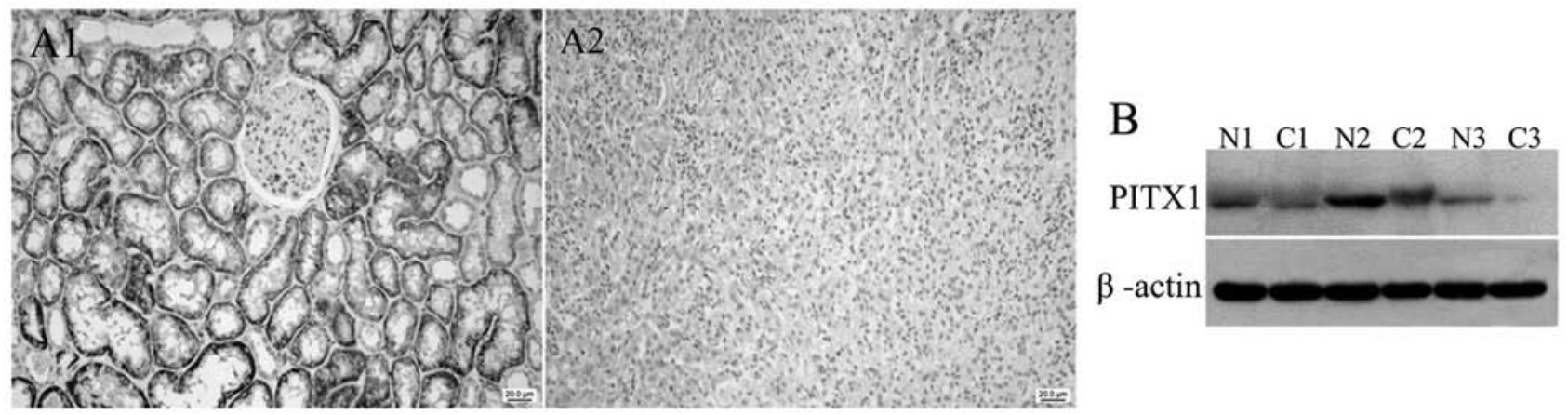

Figure 6. Protein expression of paired-like homeodomain 1 (PITX1) in clear cell renal cell carcinoma (ccRCC) and normal renal tissues by immunohistochemistry and western blot analysis. (A1) Normal renal tissues (magnification, x200). (A2) ccRCC tissues (magnification, x200). (B) Representative images of PITX1 expression in ccRCC $(\mathrm{T})$ and normal tissues $(\mathrm{N})$ by western blot analysis. 


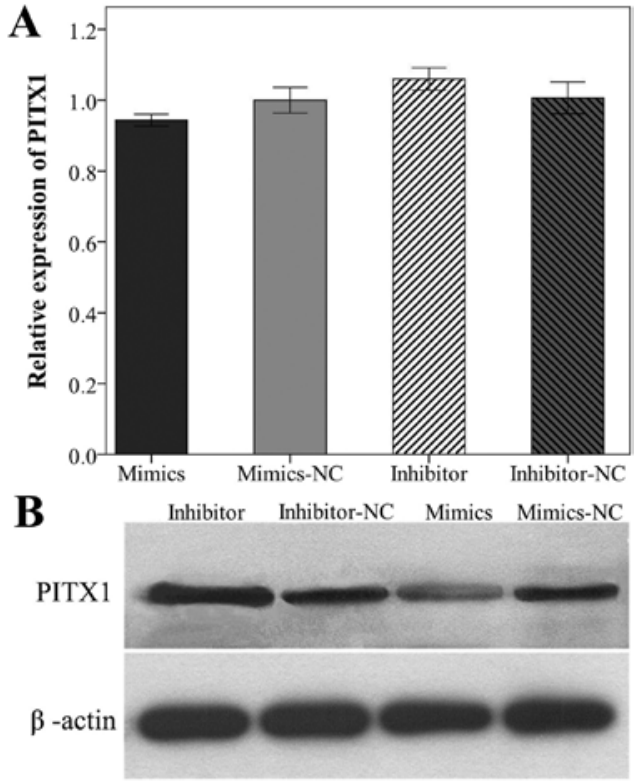

Figure 7. Regulation of paired-like homeodomain 1 (PITX1) by miR-886-3p. (A) Quantitative PCR for the PITX1 mRNA of 786-O cells following transfection with the $m i R-886-3 p$ mimics or inhibitor $(\mathrm{P}>0.05)$. Western blot analysis for PITX1 following 786-O cell transfection with $m i R-886-3 p$ mimics or inhibitor. PITX1 was significantly upregulated when 786-O cells were transfected with the miR-886-3p inhibitor and downregulated when transfected with the $m i R-886-3 p$ mimics. All the experiments were performed three times.

downregulated when transfected with the $m i R-886-3 p$ mimics $(\mathrm{P}<0.05$, Fig. 7B). These results indicated that $m i R-886-3 p$ directly regulates protein expression of PITX1 and the regulation is on post-transcriptional level.

\section{Discussion}

Since the first miRNA (miRNA-lin-4) was identified, miRNAs have been demonstrated to play a significant role in diverse cellular activities, including cell proliferation, differentiation and apoptosis $(6,26,27)$. A large number of miRNAs have been found to be aberrantly expressed in certain types of cancer and are associated with the initiation, progression, invasion and metastasis of tumors (10-12). In general, mature miRNAs bind to the 3'-UTRs of target mRNAs, leading to mRNA degradation or suppressed translation to proteins. Therefore, a specific miRNA can act as a tumor suppressor or an oncogene by inhibiting the expression of downstream oncogenes or anti-oncogenes (8). Even though potential applications of miRNAs remain restricted to experiments in laboratories, miRNAs have provided novel thinking and methods in the early diagnosis, treatment and prognosis of cancer $(28,29)$.

$m i R-886-3 p$ has been identified as a tumor suppressor in familial non-medullary thyroid cancer. The study discovered that $m i R-886-3 p$ overexpression in thyroid cancer cell lines significantly inhibited cellular proliferation, the number and size of spheroids and cellular migration and increased the number of cells in the $\mathrm{S}$ phase (8). $m i R-886-3 p$ has also been discovered to be downregulated in lung cancer. A study demonstrated that downregulated $m i R-886-3 p$ was closely correlated with a shorter survival rate of small cell lung cancer and $m i R-886-3 p$ potently repressed cell proliferation, migration and invasion of cancer cells in vitro via targeting genes, $P L K 1$ and $T G F-\beta 1$, at the post-transcription level (14). In addition, $m i R-886-3 p$ was found to be downregulated greater than 2-fold in primary squamous cell lung carcinoma (15). However, different results were observed in certain studies. A study discovered $m i R-886-3 p$ was significantly overexpressed in extranodal NK/T cell lymphoma nasal type, and considered to play crucial roles in hemopoiesis, cellular proliferation and apoptosis (16). Using miRNA microarrays, $m i R-886-3 p$ was also observed to be upregulated in malignantly transformed oral leukoplakia tissues compared to oral leukoplakia (17), indicating that $m i R-886-3 p$ may act as an oncogene in certain other cancers.

PITX1 was discovered to be downregulated in a number of types of human cancer and is correlated with a poor prognosis $(21,22,25)$. Furthermore, studies have demonstrated that PITX1 suppressed the expression of telomerase reverse transcriptase (TERT) through direct binding to the TERT promoter, thus regulating the activity of telomerase, which is crucial for cellular immortalization and cancer progression (30). In addition, PITX1 was revealed to suppress tumorigenicity by downregulating the RAS pathway through targeting a RAS guanosine triphosphate-activating factor, RASAL1 (23).

In the present study, RT-qPCR was used to validate the $m i R-886-3 p$ levels in ccRCC and adjacent normal tissues based on previous miRNAs sequencing. Consistent with the previous results, $m i R-886-3 p$ was significantly upregulated in ccRCC tissues (19). Further functional analysis revealed that forced downregulation of $m i R-886-3 p$ inhibited cell migration, proliferation and induced cell apoptosis, indicating that $m i R-886-3 p$ may be characterized as an oncogene in ccRCC. To determine the downstream genes of $m i R-886-3 p$, bioinformatics and experimental verification were performed, and PITX1 was identified. The downregulation of PITX1 in ccRCC was determined using immunohistochemistry and western blot analysis. Furthermore, the PITX1 protein, and not the mRNA, was discovered to be directly regulated by $m i R-886-3 p$, indicating that the regulation was at the post-transcriptional level. Although the specific mechanisms of PITX1 as a tumor suppressor in ccRCC remain largely unknown, $m i R-886-3 p$ was identified as an upstream regulator. The association between PITX1 expression and prognoses of ccRCC patients should be further explored, as well as the molecular mechanisms of PITX1 in the tumorigenicity, immortalization and progression of ccRCC.

The role of $m i R-886-3 p$ in cancer appears controversial as it was identified as a tumor suppressor in certain cancers and an oncogene in others $(8,14-17)$. Removing experimental error, this contradiction may be explained by the 'imperfect' complementary interactions between miRNAs and target genes. Different from siRNA, the interactions between miRNAs and 3'-UTRs of target genes are not always completely complementary (particularly in mammals), leading to the relative specifity rather than absolute specifity between miRNAs and target genes (31). Precise interactions between miRNAs and target genes may be further dictated by cell types and micro-environment, contributing to the bipolar regulations of specific miRNAs $(6,15)$.

In conclusion, to the best of our knowledge, this is the first study to reveal that $m i R-886-3 p$ was upregulated in ccRCC. 
Forced downregulation of $m i R-886-3 p$ inhibited cellular migration, proliferation and induced cell apoptosis by targeting the tumor suppressor PITXI in ccRCC.

\section{Acknowledgements}

The present study was supported by the National Natural Science Foundation of China (grant no. 81101922), Medical Scientific Research Foundation of Guangdong Province of China (grant no. A2012584 and A2013606) and Science and Technology Development Fund Project of Shenzhen (grant no. JCYJ20130402114702124).

\section{References}

1. Jemal A, Siegel R, Xu J and Ward E: Cancer statistics, 2010. CA Cancer J Clin 60: 277-300, 2010.

2. Patel C, Ahmed A and Ellsworth P: Renal cell carcinoma: a reappraisal. Urol Nurs 32: 182-191, 2012.

3. Rouvière $\mathrm{O}$, Bouvier R, Négrier $\mathrm{S}$, Badet $\mathrm{L}$ and Lyonnet $\mathrm{D}$ : Nonmetastatic renal-cell carcinoma: is it really possible to define rational guidelines for post-treatment follow-up? Nat Clin Pract Oncol 3: 200-213, 2006.

4. McLaughlin JK, Lipworth L and Tarone RE: Epidemiologic aspects of renal cell carcinoma. Semin Oncol 33: 527-533, 2006.

5. Cairns P: Renal cell carcinoma. Cancer Biomark 9: 461-473, 2010.

6. Jiang L, Liu X, Chen Z, et al: MicroRNA-7 targets IGF1R (insulin-like growth factor 1 receptor) in tongue squamous cell carcinoma cells. Biochem J 432: 199-205, 2010.

7. Soeda S, Ohyashiki JH, Ohtsuki K, et al: Clinical relevance of plasma miR-106b levels in patients with chronic obstructive pulmonary disease. Int J Mol Med 31: 533-539, 2013.

8. Xiong Y, Zhang L, Holloway AK, et al: miR-886-3p regulates cell proliferation and migration, and is dysregulated in familial non-medullary thyroid cancer. PLoS One 6: e24717, 2011.

9. Rosén A, Bergh AC, Gogok P, et al: Lymphoblastoid cell line with B1 cell characteristics established from a chronic lymphocytic leukemia clone by in vitro EBV infection. Oncoimmunology 1: 18-27, 2012.

10. Shibuya H, Iinuma H, Shimada R, Horiuchi A and Watanabe T: Clinicopathological and prognostic value of microRNA-21 and microRNA-155 in colorectal cancer. Oncology 79: 313-320, 2010.

11. Akao Y, Noguchi S, Iio A, et al: Dysregulation of microRNA-34a expression causes drug-resistance to 5-FU in human colon cancer DLD-1 cells. Cancer Lett 300: 197-204, 2011.

12. Tili E, Michaille JJ, Wernicke D, et al: Mutator activity induced by microRNA-155 (miR-155) links inflammation and cancer. Proc Natl Acad Sci USA 108: 4908-4913, 2011

13. Kong X, Li G, Yuan Y, et al: MicroRNA-7 inhibits epithelial-to-mesenchymal transition and metastasis of breast cancer cells via targeting FAK expression. PLoS One 7: e41523, 2012.

14. Liu G, Keeler BE, Zhukareva V and Houlé JD: Cycling exercise affects the expression of apoptosis-associated microRNAs after spinal cord injury in rats. Exp Neurol 226: 200-206, 2010.
15. Kharaziha P, Ceder S, Li Q and Panaretakis T: Tumor cell-derived exosomes: a message in a bottle. Biochim Biophys Acta 1826: 103-111, 2012.

16. Xu F, Zhang X, Lei Y, et al: Loss of repression of HuR translation by miR-16 may be responsible for the elevation of HuR in human breast carcinoma. J Cell Biochem 111: 727-734, 2010.

17. Lennox KA and Behlke MA: A direct comparison of anti-microRNA oligonucleotide potency. Pharm Res 27: 1788-1799, 2010.

18. Wang Z, Zhang J, Luo $\mathrm{H}$, et al: Screening and confirmation of microRNA markers for forensic body fluid identification. Forensic Sci Int Genet 7: 116-123, 2013.

19. Li X, Chen J, Hu X, et al: Comparative mRNA and microRNA expression profiling of three genitourinary cancers reveals common hallmarks and cancer-specific molecular events. PLoS One 6: e22570, 2011.

20. Zhou L, Chen J, Li Z, et al: Integrated profiling of microRNAs and mRNAs: microRNAs located on Xq27.3 associate with clear cell renal cell carcinoma. PLoS One 5: e15224, 2010.

21. Chen Y, Knosel T, Ye F, Pacyna-Gengelbach M, Deutschmann N and Petersen I: Decreased PITX1 homeobox gene expression in human lung cancer. Lung Cancer 55: 287-294, 2007.

22. Chen YN, Chen H, Xu Y, Zhang X and Luo Y: Expression of pituitary homeobox 1 gene in human gastric carcinogenesis and its clinicopathological significance. World J Gastroenterol 14: 292-297, 2008.

23. Kolfschoten IG, van Leeuwen B, Berns K, et al: A genetic screen identifies PITX1 as a suppressor of RAS activity and tumorigenicity. Cell 121: 849-858, 2005

24. Qi DL, Ohhira T, Fujisaki C, et al: Identification of PITX1 as a TERT suppressor gene located on human chromosome 5. Mol Cell Biol 31: 1624-1636, 2011.

25. Knosel T, Chen Y, Hotovy S, Settmacher U, AltendorfHofmann A and Petersen I: Loss of desmocollin 1-3 and homeobox genes PITX1 and CDX2 are associated with tumor progression and survival in colorectal carcinoma. Int J Colorectal Dis 27: 1391-1399, 2012.

26. Zhai Q, Zhou L, Zhao C, et al: Identification of miR-508-3p and miR-509-3p that are associated with cell invasion and migration and involved in the apoptosis of renal cell carcinoma. Biochem Biophys Res Commun 419: 621-626, 2012.

27. Liu M, Tang Q, Qiu M, et al: miR-21 targets the tumor suppressor RhoB and regulates proliferation, invasion and apoptosis in colorectal cancer cells. FEBS Lett 585: 2998-3005, 2011.

28. Wang GK, Zhu JQ, Zhang JT, et al: Circulating microRNA: a novel potential biomarker for early diagnosis of acute myocardial infarction in humans. Eur Heart J 31: 659-666, 2010.

29. Guo LJ and Zhang QY: Decreased serum miR-181a is a potential new tool for breast cancer screening. Int J Mol Med 30: 680-686, 2012.

30. Sendt W, Rippe V, Flor I, Drieschner N and Bullerdiek J: Monosomy and ring chromosome 13 in a thyroid nodular goiter-do we underestimate its relevance in benign thyroid lesions? Cancer Genet 205: 128-130, 2012.

31. Kim VN: MicroRNA biogenesis: coordinated cropping and dicing. Nat Rev Mol Cell Biol 6: 376-385, 2005.

32. Guinan P, Sobin LH, Algaba F, et al: TNM staging of renal cell carcinoma: Workgroup No. 3. Union International Contre le Cancer (UICC) and the American Joint Committee on Cancer (AJCC). Cancer 80: 992-993, 1997. 